Jan van Meerveld

Received: 26 November 2003

Accepted: 15 January 2004

Published online: 20 March 2004

(c) Springer-Verlag 2004

J. van Meerveld

Institute of Polymers, Department of

Materials, ETH-Zürich, ETH-Zentrum,

Zürich, Switzerland

E-mail: meerveld@mat.ethz.ch

\section{A method to extract the monomer friction coefficient from the linear viscoelastic behavior of linear, entangled polymer melts}

with values of $\zeta$ reported in the literature for linear, non-entangled polymer melts. It is observed that for isotactic and atactic polypropylene $M_{\mathrm{e}}$ differs by a factor 1.25 depending on the approach taken. As a consequence, the magnitude of $\tau_{\mathrm{e}}$ and $\zeta$ differ by a factor of about 3.0 and 1.8 , respectively. The knowledge of $\tau_{\mathrm{e}}$ (or $\zeta$ ) is of importance in order to obtain a better understanding of flow induced crystallization experiments on iPP.

Keywords Isotactic polypropylene · Atactic polypropylene .

Linear polymer melts .

Linear viscoelasticity

Monomer friction coefficient

\section{Introduction}

The rheological relevant time scales, as the reptation and longest Rouse time, can be directly related to the molecular weight (distribution) of the melt using molecular theories based on the tube concept (Doi and Edwards 1986). Alternatively, the rheological response influences the flow induced crystallization dynamics and consequently the semi-crystalline morphology of the material. In particular, the transition from the spherulitic to the so-called 'shish-kebab' morphology changes the solid-state properties of the material (Keller and Kolnaar 1997). The development of the shish-kebab morphology is generally believed to result from chain stretching of the high molecular weight, HMW, chains in the high end tail of the molecular weight distribution of the melt (Vleeshouwers and Meijer 1996; Keller and Kolnaar 1997; Nogales et al. 2001; Seki et al. 2002). This implies that for the HMW chains the Deborah number based on the longest Rouse time, $\tau_{R}, D e_{s}=\tau_{R} \dot{\gamma}$ or $\tau_{R} \dot{\varepsilon}$ is larger than 1, with $\dot{\gamma}$ and $\dot{\varepsilon}$ the shear and extensional rate respectively. The magnitude of $\tau_{R}$ is related to the molecular weight, $M$, of the chains via (Doi and Edwards 1986; Larson et al. 2003)

$\tau_{R}=\tau_{\mathrm{e}}\left(M / M_{\mathrm{e}}\right)^{2}$.

The knowledge of $M_{\mathrm{e}}$ and the equilibration time $\tau_{e}$ (which is the Rouse relaxation time of a chain of length equal to one tube segment) is thus sufficient to determine $\tau_{R}$ directly from the molecular weight. (The magnitude of $\tau_{e}$ is directly related to the monomeric friction coefficient, $\zeta$ (Ferry 1980; Larson et al. 2003).) This 
approach is advantageous as it is difficult, if not impossible, to obtain $\tau_{R}$ for the HMW chains experimentally from the linear viscoelastic regime (van Meerveld et al. 2003). In addition, it is likely that the stress contribution from the high-end tail is 'screened' by the remaining part of the MWD in shear flows (Graham et al. 2001). The drawback is that, to the best of my knowledge, $\tau_{\mathrm{e}}$ or $\zeta$ is not reported in the literature for iPP, which is a popular material for flow induced crystallization experiments. This paper has the goal to obtain the magnitude of $\tau_{\mathrm{e}}$ and $\zeta$ for iPP, as this is helpful to obtain a better understanding of the observations in flow induced crystallization experiments of iPP.

In the next section, two procedures are described to obtain $\tau_{\mathrm{e}}$ from molecular based theories for linear, entangled polymer melts. In the following section these two procedures are evaluated for different polymer systems and the resulting magnitudes of $\zeta$ are compared with data reported in the literature, which are obtained for linear, non-entangled polymer melts. One procedure is applied to iPP and aPP in the section after that. Finally conclusions are drawn in the last section.

\section{Theory}

Definition of the molecular weight per entanglement from experiments

Before addressing the two procedures to obtain $\tau_{\mathrm{e}}$ the different definitions of the molecular weight per entanglement, $M_{\mathrm{e}}$, are introduced, where we follow the notation as used in Larson et al. (2003). The magnitude of $M_{\mathrm{e}}$ follows from the expression according to Ferry (1980):

$M_{\mathrm{e}}^{F}=\frac{\rho R T}{G_{N}^{0}}$

or that proposed by Fetters et al. (1994):

$M_{\mathrm{e}}^{G}=\frac{4}{5} \frac{\rho R T}{G_{N}^{0}}$,

with $R$ the universal gas constant, $T$ the absolute temperature, $\rho$ the density and $G_{N}^{0}$ the plateau modulus, which is accessible experimentally. For monodisperse melts the magnitude of $G_{N}^{0}$ can be determined by, first, integrating the area under the loss modulus vs frequency, $G^{\prime \prime}$ vs $\omega$, plot (Ferry 1980), second, from creep experiments (Plazek and Plazek 1983), or third by using the phenomenological relationship, $G_{N}^{0}=3.56 G^{\prime \prime}{ }_{\max }$ (Fetters et al. 1994, 1996, 1999).

\section{Procedure A}

The first procedure to determine $\tau_{e}$ is based on the fit of the storage modulus, $G^{\prime}(\omega)$, and loss modulus, $G^{\prime \prime}(\omega)$, by the molecular based model developed by Milner and McLeish (1998), the improved version by Likhtman and McLeish (2002), the LM-model, and the dual constraint model of Pattamaprom et al. (2000) and Pattamaprom and Larson (2001), the DC model. In this section only the relevant differences between the DC and LM model are discussed for the current purpose and the reader is referred to the original papers for the model formulations. The $\tau_{\mathrm{e}}($ or $\zeta)$ and $M_{\mathrm{e}}$ are the only adjustable parameters in both models. However, the DC model is based on $M_{\mathrm{e}}^{F}$ and the LM model on $M_{\mathrm{e}}^{G}$. The difference between considering $M_{\mathrm{e}}^{F}$ and $M_{\mathrm{e}}^{G}$ affects, first, the number of entanglements, second, the numerical prefactor of $\tau_{\mathrm{e}}$ and, third, the numerical prefactor of the time scale of 'early time' primitive path fluctuations, $\tau_{\text {early }}$, as discussed in detail by Larson et al. (2003). The resulting differences between the LM and DC model are given in Table 1. (The significance of the tube length fluctuations changes in a non-linear fashion due to the combined effect of the differences on the time scales given in Table 1.) In addition two differences between the DC and LM model are of relevance. First, the ratio of the reptation time without tube length fluctuation, $\tau_{d}^{0}$, to the longest Rouse time, $\tau_{R}$, is equal to $\tau_{d}^{0} / \tau_{R}=3 Z$ in the LM model, whereas in the DC model $\tau_{d}^{0} / \tau_{R}=6 Z$. Second, the description of the Rouse dynamics is different. In particular, the formulation of the Rouse dynamics in the LM model shows that $1 / 5$ of the stress relaxes through 'longitudinal relaxation modes' in the terminal regime. The LM model thus naturally accounts for the numerical prefactor $4 / 5$, which appears in the definition of $M_{\mathrm{e}}^{G}$, Eq. (3). Consequently the modulus in the LM model is defined as $G^{\mathrm{LM}}=\rho R T / M_{\mathrm{e}}^{G}$, which is a factor $5 / 4$ larger than the experimentally measured value of $G_{N}^{0}$, i.e., $G^{\mathrm{LM}}=5 G_{N}^{0} / 4$. As a result of the differences in the DC and LM model the magnitude of $\tau_{\mathrm{e}}$ is not identical

Table 1 Specification of the model parameters in the model of Likhtmann and McLeish (2002) and the Dual-Constraint model (Pattamaprom et al. 2000): the molecular weight per entanglement, $M_{\mathrm{e}}$, the modulus, $G$, the number of entanglements, $Z$, the equilibration time, $\tau_{\mathrm{e}}$, the time scale for 'early time' primitive path fluctuations, $\tau_{\text {early }}$, and the ratio of the longest Rouse time, $\tau_{R}$, to the reptation time without tube length fluctuations, $\tau_{d}^{0}$. It is noted that $\tau_{\text {early }}$ is not implemented in the LM model but resolved exactly by full chain simulations (Likhtman and McLeish 2002)

\begin{tabular}{lll}
\hline Model & $\begin{array}{l}\text { Likhtmann } \\
\text { and McLeish }\end{array}$ & Dual-constraint \\
\hline$M_{\mathrm{e}}$ & $M_{\mathrm{e}}^{G}$ & $M_{\mathrm{e}}^{F}$ \\
$G$ & $\frac{\rho R T}{M_{\mathrm{e}}^{G}}$ & $\frac{\rho R T}{M_{e}^{F}}$ \\
$Z$ & $M / M_{\mathrm{e}}^{G}$ & $M / M_{\mathrm{e}}^{F}$ \\
$\tau_{\mathrm{e}}$ & $\left(\frac{M_{e}^{G}}{M_{0}}\right)^{2} \frac{\zeta b^{2}}{3 \pi^{2} k_{b} T}$ & $\left(\frac{M_{\mathrm{e}}^{F}}{M_{0}}\right)^{\frac{\zeta b^{2}}{3 \pi^{2} b_{T} T}}$ \\
$\tau_{\text {early }}$ & $\frac{9}{16 \pi^{3}} \tau_{\mathrm{e}} Z^{4} s^{4}$ & $\frac{225}{25 \sigma^{3}}\left(\tau_{\mathrm{e}} / 2\right)(Z / 2)^{4} s^{4}$ \\
$\tau_{R}$ & $\tau_{\mathrm{e}} Z^{2}$ & $\tau_{\mathrm{e}} Z^{2} / 2$ \\
$\tau_{\mathrm{d}}^{0} / \tau_{R}$ & $3 Z$ & $6 Z$ \\
\hline
\end{tabular}


and therefore denoted as $\tau_{\mathrm{e}}^{\mathrm{DC}}$ and $\tau_{\mathrm{e}}^{\mathrm{LM}}$ respectively. From experiments it is observed that $2 \tau_{\mathrm{e}}^{\mathrm{LM}} \approx \tau_{\mathrm{e}}^{\mathrm{DC}}$. In general it is possible to shift from the DC to the LM model by taking into account a correction factor of $5 / 4$, or powers thereof, and using the experimentally observed relationship $2 \tau_{\mathrm{e}}^{\mathrm{LM}} \approx \tau_{\mathrm{e}}^{\mathrm{DC}}$, see Table 2 . The advantage of this procedure is that a good description of $G^{\prime}(\omega)$ and $G^{\prime \prime}(\omega)$ in the terminal, the intermediate and the glassy regime requires a correct magnitude of both $\tau_{\mathrm{e}}$ and $M_{\mathrm{e}}$.

\section{Procedure B}

For different reasons $G^{\prime}(\omega)$ and $G^{\prime \prime}(\omega)$ cannot be determined experimentally from the terminal to the glassy regime for melts of certain polymers. For example due to, first, the limited benefit from the time-temperature superposition principle (Kraft et al. 1999), second, a small temperature window due to a 'high' crystallization temperature and the onset of degradation at relative 'low' temperatures or, third, the difficulty to synthesize a melt of long monodisperse chains (Eckstein et al. 1998). In many cases only the terminal regime is accessible and the zero shear rate viscosity as a function of the weight averaged molecular weight, $M_{w}$, can be determined.

In procedure $\mathrm{B} M_{\mathrm{e}}^{\mathrm{G}}$, or $M_{\mathrm{e}}^{\mathrm{F}}$, is taken from the literature (Fetters et al. 1994, 1996, 1999) and the magnitude of $\tau_{e}$ is determined from the zero shear rate viscosity, $\eta_{0}$, as predicted by the DC and LM model. Actually, this approach is similar in spirit as reported by Ferry (1980). The important difference is that in the current approach the effect of tube length fluctuations is incorporated in the DC and LM model, which are essential to predict the scaling $\eta_{0} \sim Z^{3.4}$.

Pattamaprom and Larson (2001) performed a mapping of the double reptation model (Tsenoglou 1987; des Cloizeaux 1988, 1990) on the predicted $\eta_{0}$ of the DC model. Doing so they arrive at the following expression between $\eta_{0}$ and $\tau_{e}$ :

Table 2 The magnitude of the modulus $G$, the molecular weight per entanglement, $M_{\mathrm{e}}$, and the equilibration time, $\tau_{\mathrm{e}}$, for different polymers following from the different procedures. The magnitudes for A-LM are reported in Likhtman and McLeish (2002), or ob-
$\eta_{0}\left(M / M_{\mathrm{e}}^{\mathrm{F}}\right)=0.051 \tau_{\mathrm{e}}^{\mathrm{DC}} G_{\mathrm{N}}^{0}\left(M / M_{\mathrm{e}}^{\mathrm{F}}\right)^{3.4}$,

where $M_{\mathrm{e}}^{\mathrm{F}}$ is used for consistency reasons. A similar exercise for the LM model, with the constraint release parameter $c_{v}$ equal to one, $c_{v}=1.0$, (Likhtman and McLeish 2002) gives

$\eta_{0}\left(M / M_{\mathrm{e}}^{G}\right)=0.064 \tau_{\mathrm{e}}^{L M} \frac{\rho R T}{M_{\mathrm{e}}^{G}}\left(M / M_{\mathrm{e}}^{G}\right)^{3.4}$,

again using $M_{\mathrm{e}}^{G}$ to be consistent. It is noted that the numerical prefactor in Eq. (5), and consequently the magnitude of $\tau_{e}$ resulting from Eq. (5), depends on the magnitude of $c_{v}$.

For many polymers the $\eta_{0}$ as a function of $M_{w}$ is available, which is represented by the phenomenological expression of the form

$\eta_{0}\left(M_{w}\right)=\alpha M_{w}^{\beta}$,

where the magnitude of $\alpha$ and $\beta$ are given in Table 3, which is observed to hold for both mono- and polydisperse melts (Struglinski and Graessley 1985; Berger and Meissner 1992; Aguliar et al. 2003; Vega et al. 2003) in agreement with predictions of the DC model (Pattamaprom and Larson 2001). Observation of Table 3 illustrates that in a number of studies the exponent $\beta$ equals the theoretical value of 3.4 , but sometimes $\beta \approx 3$.6. It should be noted that for $\beta \approx 3.6$ the magnitude of $\alpha$ is roughly an order of magnitude smaller compared to $\beta=3.4$ for both PE and aPP. In the next two sections only the expressions with $\beta=3.4$ are used, which are consistent with Eqs. (4) and (5).

\section{Application of procedure A and B, and comparison with data in the literature}

In this section the magnitude of $\tau_{\mathrm{e}}$ and $\zeta$ are determined for polyethylene, PE, hydrogenated polybutadiene,

tained in the section 'Application of procedure A and B, and comparison with data in the literature', and for A-DC reported in Pattamaprom et al. (2000) and Pattamaprom and Larson (2001). $\mathrm{n} / \mathrm{a}$ denotes not available

\begin{tabular}{lllllllll}
\hline & Unit & Procedure & PE & PI & h-PBD & 1,4-PBD & aPP \\
\hline$T$ & K & & 463 & 298 & 463 & 301 & 348 \\
$G$ & $\mathrm{kPa}$ & A-LM & 3250 & 536 & 2888 & 1840 & 757 \\
$M_{\mathrm{e}}$ & $\mathrm{g} / \mathrm{mol}$ & A-LM & 860 & 4160 & 1000 & 1930 & 3150 \\
$\tau_{\mathrm{e}}$ & $\mathrm{s}$ & A-LM & n $/ \mathrm{a}$ & $3.0 \times 10^{-5}$ & $3.5 \times 10^{-9}$ & $4.9 \times 10^{-7}$ & $5.7 \times 10^{-6}$ & 9.269 \\
$\tau_{\mathrm{e}}$ & $\mathrm{s}$ & B-LM & $1.45 \times 10^{-9}$ & n/a & $2.87 \times 10^{-9}$ & $4.56 \times 10^{-7}$ & $5.3 \times 10^{-6}$ & $8.21 \times 10^{-4}$ \\
$G$ & {$[\mathrm{kPa}]$} & A-DC & 2600 & 434 & 2310 & 1250 & $\mathrm{n} / \mathrm{a}$ \\
$M_{\mathrm{e}}$ & $\mathrm{g} / \mathrm{mol}$ & A-DC & 1035 & 5200 & 1250 & 2268 & 200 \\
$\tau_{\mathrm{e}}$ & $\mathrm{s}$ & A-DC & $7.0 \times 10^{-9}$ & $6.0 \times 10^{-5}$ & $7.0 \times 10^{-9}$ & $1.51 \times 10^{-6}$ & $\mathrm{n} / \mathrm{a}$ & $\mathrm{n} / \mathrm{a}$ \\
$\tau_{\mathrm{e}}$ & $\mathrm{s}$ & B-DC & $4.429 \times 10^{-9}$ & n/a & $9.23 \times 10^{-9}$ & $1.46 \times 10^{-6}$ & $\mathrm{n} / \mathrm{a}$ \\
\hline
\end{tabular}


Table 3 Reported values of the parameters $\alpha$ and $\beta$ in the expression $\eta_{0}=\alpha M_{w}^{\beta}$ for different polymers at a temperature $T$

\begin{tabular}{lllcc}
\hline Material & Reference & $\alpha \times 10^{-15}$ & $\beta$ & $T$ \\
& Units & $\mathrm{Ns} \mathrm{mol}^{\beta} / \mathrm{g}^{\alpha}$ & - & $K$ \\
\hline PE & Pearson et al. (1987) & 3.76 & 3.64 & 448 \\
PE & Wood-Adams et al. (2000) & 3.40 & 3.6 & 463 \\
PE & Aguilar et al. (2001) & 31.7 & 3.41 & 463 \\
h-PBD & Pattamaprom and Larson (2001) & 30 & 3.41 & 463 \\
1.4-PBD & Struglinksi and Graessley (1985) & 363 & 3.41 & 298 \\
aPP & Aguilar et al. (2003) & 340 & 3.40 & 348 \\
aPP & Pearson et al. (1988) & 53.4 & 3.59 & 348 \\
iPP & Wasserman and Graessley (1996) & 5.32 & 3.60 & 463 \\
PS & Wasserman and Graessley (1992) & 16800 & 3.4 & 423 \\
PS & Graessley and Roovers (1979) & 97.7 & 3.41 & 442.5 \\
PIB & Fetters et al. (1991) & 4690 & 3.43 & 298 \\
\hline
\end{tabular}

h-PBD, 1,4-polybutadiene, 1,4-PBD, atactic polypropylene, aPP, polyisoprene, PI, and polyisobutylene, PIB, and polystyrene, PS, using procedure $\mathrm{A}$ in the first subsection, and procedure $\mathrm{B}$ in the second subsection. In the third subsection the magnitude of $\zeta$ following from procedures $\mathrm{A}$ and $\mathrm{B}$ are compared with values reported in the literature for linear, non-entangled polymer melts.

Procedure A for the DC and LM model

The DC model has been compared to experimental results of mono- and polydisperse PS, h-PBD, 1,4-PBD, PI and PE melts in Pattamaprom et al. (2000) and Pattamaprom and Larson (2001), whereas the LM model is only compared to experiments on nearly monodisperse PS and 1,4-PBD in Likhtman and McLeish (2002). In order to perform the analysis for a larger number of polymer systems the LM model, with $c_{v}=1$, is used to describe $G^{\prime}(\omega)$ and $G^{\prime \prime}(\omega)$ of the nearly monodisperse h-PBD (Raju et al. 1979), PI (Fetters et al. 1993), and aPP (Pearson et al. 1988). Good agreement between the predictions of the LM model and the experimental results for PI (Fetters et al. 1993) and h-PBD (Raju et al. 1979) are obtained using $\tau_{\mathrm{e}}^{\mathrm{LM}}=0.5 \tau_{\mathrm{e}}^{\mathrm{DC}}$ and taking $M_{\mathrm{e}}^{G}$ from the literature; see Figs. 1 and 2 and Table 2. (In contrast to PS, PI, and h-PBD one observes that for 1,4PBD $3 \tau_{\mathrm{e}}^{\mathrm{LM}} \approx \tau_{\mathrm{e}}^{\mathrm{DC}}$. It should be noted that the magnitudes of $G^{\mathrm{LM}}$ and $M_{\mathrm{e}}^{G}$ of the LM model are adjusted independently in order to optimize the description of the 1,4-PBD melt, which is discussed in detail by Likhtmann and McLeish (2002).) For $\rho=825 \mathrm{~kg} / \mathrm{m}^{3}$ (Fetters et al. 1994), $M_{\mathrm{e}}^{G}=3150 \mathrm{~g} / \mathrm{mol}$ and $\tau_{\mathrm{e}}=5.7 \times 10^{-6} \mathrm{~s}$ the linear viscoelastic response of aPP at $T=348 \mathrm{~K}$ (Pearson et al. 1988 ) is correctly predicted by the LM model; see Fig. 3. However, the magnitude of $M_{\mathrm{e}}^{G}$ is about a factor 1.25 smaller compared to $M_{\mathrm{e}}^{G}=4000 \mathrm{~g} / \mathrm{mol}$, which is obtained after a linear interpolation between the magnitudes reported at $T=298 \mathrm{~K}$ and $T=413 \mathrm{~K}$ in Fetters et al. $(1994,1996)$. For $M_{\mathrm{e}}^{G}=3150 \mathrm{~g} / \mathrm{mol}$ one obtains $G^{\mathrm{LM}}=757 \mathrm{kPa}$, which corresponds to $G_{N}^{0}=606 \mathrm{kPa}$. (The magnitude of $G_{N}^{0}$ deviates considerably from the

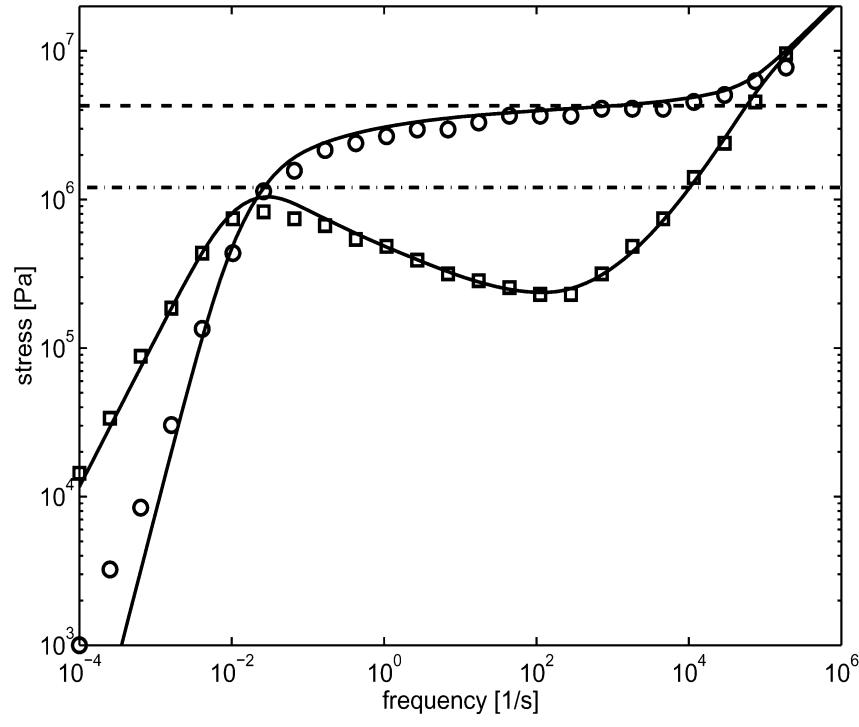

Fig. 1 The linear viscoelastic response of nearly monodisperse polyisoprenes, PI, at $T=298 \mathrm{~K}$. Predictions of the LM model (full lines). Experimental data are taken from Fetters et al. (1993). The dashed line indicates $4 G^{\mathrm{LM}} / 5$ and the dash-dotted line the magnitude of $G_{\text {max }}^{\prime \prime}$ according to $3.56 G^{\prime \prime}{ }_{\text {max }}=4 G^{\mathrm{LM}} / 5$

scattering in Figs. 1 and 2 of Fetters et al. (1994).) The chain characteristics and $M_{\mathrm{e}}^{G}$ of deuterated head-to-head polypropylene, dhhPP, are nearly identical to that of aPP (Fetters et al. 1994, 1999; Krishnamoorti et al. 2002). Therefore the description of $G^{\prime}(\omega)$ and $G^{\prime \prime}(\omega)$ of the dhhPP melt at $T=323 \mathrm{~K}$ reported by Gell et al. (1997) is also fitted by the LM model to investigate if the deviation observed for the aPP melts is also found for the dhhPP melt. For $\rho=922 \mathrm{~kg} / \mathrm{m}^{3}$ (Gell et al. 1997), $M_{\mathrm{e}}^{G}=2600 \mathrm{~g} / \mathrm{mol}$ and $\tau_{\mathrm{e}}=2.6 \times 10^{-4} \mathrm{~s}$ the LM model correctly describes $G^{\prime}(\omega)$ and $G^{\prime \prime}(\omega)$; see Fig. 4. Similar to the aPP melts, a good description of $G^{\prime}(\omega)$ and $G^{\prime \prime}(\omega)$ of the dhhPP melt by the LM model requires one to take $M_{\mathrm{e}}^{G}$ smaller compared to the experimental value of $M_{\mathrm{e}}^{G}=4360 \mathrm{~g} / \mathrm{mol}$. In the case of dhhPP the difference is a factor 1.67. (The magnitude of $M_{\mathrm{e}}^{G}$ is the average of the three dhhPP melts investigated by Gell et al. (1997), 


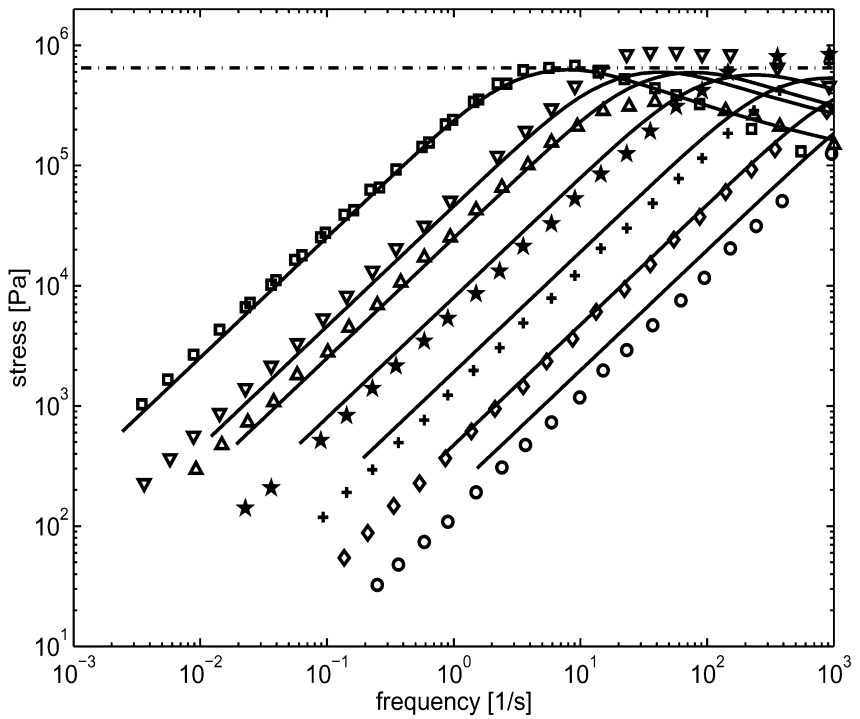

Fig. 2 The linear viscoelastic response of nearly monodisperse hydrogenated polybutadienes, h-PBD, at $T=463 \mathrm{~K}$. Predictions of the LM model (full lines). Experimental data are taken from Raju et al. (1979) for $M_{w}=41.7 \mathrm{~kg} / \mathrm{mol}$ (open circles), $M_{w}=53.3 \mathrm{~kg} / \mathrm{mol}$ (open diamonds), $M_{w}=80.3 \mathrm{~kg} / \mathrm{mol}$ (crosses), $M_{w}=123 \mathrm{~kg} / \mathrm{mol}$ (stars), $M_{w}=174 \mathrm{~kg} / \mathrm{mol}$ (open triangles), $M_{w}=211 \mathrm{~kg} / \mathrm{mol}$ (inverted open triangles), $M_{w}=360 \mathrm{~kg} / \mathrm{mol}$ (open squares). The dashdotted line denotes the magnitude of $G^{\prime \prime}$ max according to $3.56 G^{\prime \prime} \max =4 G^{\mathrm{LM}} / 5$

which follow from Eq. (3) using the reported values for $G_{N}^{0}$ and $\rho$ by Gell et al. (1997).) It should be noted that for the aPP samples and dhhPP sample $G_{N}^{0} J_{e}^{0} \geq 4$ (Pearson et al. 1988; Gell et al. 1997) whereas $G_{N}^{0} J_{e}^{0}$ is in general observed to be around 2.0-2.2 for monodisperse samples (Raju et al. 1981). This is believed to originate from a high molecular weight tail in the molecular weight distribution (Pearson et al. 1988; Gell et al. 1997).

\section{Procedure B for the DC and LM model}

For the application of procedure B the experimental relationship for $\eta_{0}\left(M_{w}\right)$ is taken from experiments on polydisperse melts. This is opposite to procedure A which can only be applied to monodisperse systems, at least for the LM model. To be consistent identical magnitudes of $M_{\mathrm{e}}^{F}$ and $M_{\mathrm{e}}^{G}$ are taken to determine $\tau_{\mathrm{e}}^{\mathrm{DC}}$ and $\tau_{\mathrm{e}}^{\mathrm{LM}}$ from Eqs. (4) and (5) respectively. The resulting magnitudes of $\tau_{\mathrm{e}}^{\mathrm{DC}}$ and $\tau_{\mathrm{e}}^{\mathrm{LM}}$ are given in Table 2. In general the magnitudes of $\tau_{\mathrm{e}}^{\mathrm{DC}}$ and $\tau_{\mathrm{e}}^{\mathrm{LM}}$ of procedures $\mathrm{A}$ and $\mathrm{B}$ differ by less than a factor 1.35 , except for PE where a factor of 2 difference is observed. The good agreement is partly expected as the description of $G^{\prime}(\omega)$ and $G^{\prime \prime}(\omega)$ in the terminal region, where $G^{\prime}(\omega) \sim \omega^{-2}$ and $G^{\prime \prime}(\omega) \sim \omega^{-1}$, by the DC and LM model is closely related to the predicted $\eta_{0}$.

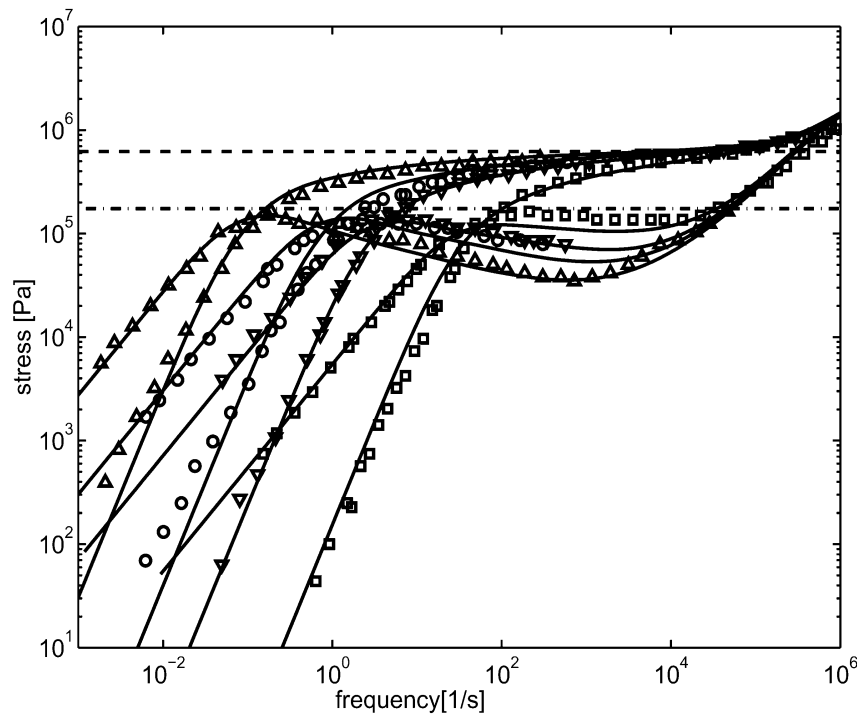

Fig. 3 The linear viscoelastic response of nearly monodisperse atactic polypropylene, aPP, at $T=348 \mathrm{~K}$. Predictions of the LM model (full lines). Experimental data are taken from Pearson et al (1988) for $M_{w}=63.5 \mathrm{~kg} / \mathrm{mol}$ (open squares), $M_{w}=126 \mathrm{~kg} / \mathrm{mol}$ (inverted open triangles), $M_{w}=189 \mathrm{~kg} / \mathrm{mol}$ (open circles), $M_{w}=371 \mathrm{~kg} / \mathrm{mol}$ (open triangles). The dashed line indicates $4 G^{\mathrm{LM}} / 5$ and the dash-dotted line the magnitude of $G^{\prime \prime}{ }_{\max }$ according to $3.56 G^{\prime \prime}{ }_{\max }=4 G^{\mathrm{LM}} / 5$

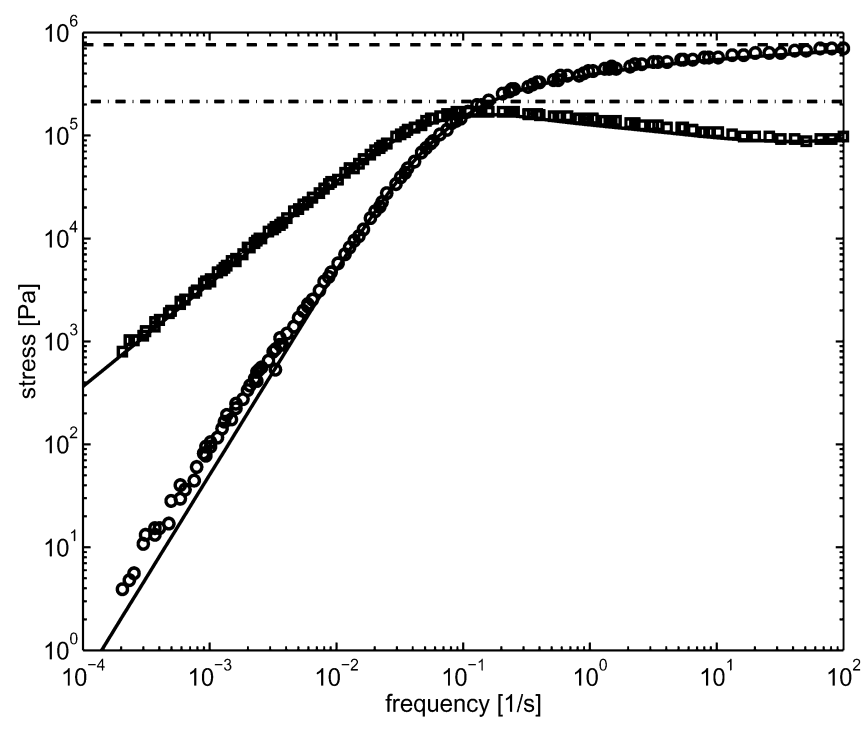

Fig. 4 The linear viscoelastic response of nearly monodisperse deuterated head-to-head polypropylene, dhhPP, at $T=323 \mathrm{~K}$. Predictions of the LM model (full lines). Experimental data (symbols) are taken from Gell et al. (1997). The dashed line indicates $4 G^{\mathrm{LM}} / 5$ and the dash-dotted line the magnitude of $G^{\prime \prime}$ max according to $3.56 G^{\prime \prime}{ }_{\max }=4 G^{\mathrm{LM}} / 5$

\section{Comparison with other methods}

Finally, the magnitude of $\zeta$, which is directly related to $\tau_{\mathrm{e}}$, is compared with values of $\zeta$ reported in the 
Table 4 Magnitude of the monomer friction coefficient $\zeta$ for different polymers obtained from procedure A of the LM model, A-LM, procedure B of the LM model, B-LM, using Eq. 7. Magnitudes of $<R^{2}>_{0} / M$ are taken from Fetters et al. (1994, 1996, 1999)

\begin{tabular}{lllll}
\hline Material & $\mathrm{T}$ & $\begin{array}{l}\zeta: \mathrm{A}-\mathrm{LM} \\
\mathrm{Ns} / \mathrm{m}\end{array}$ & $\begin{array}{l}\zeta: \mathrm{B}-\mathrm{LM} \\
\mathrm{Ns} / \mathrm{m}\end{array}$ & $\begin{array}{l}\zeta: \text { literature } \\
\text { Ns } / \mathrm{m}\end{array}$ \\
\hline PE & $\mathrm{K}$ & $\mathrm{n} / \mathrm{a}$ & $4.15 \times 10^{-13}$ & $4.74 \times 10^{-13 \mathrm{a}}$ \\
PI & 463 & $4.13 \times 10^{-10}$ & $\mathrm{n} / \mathrm{a}$ & $3.26 \times 10^{-10 \mathrm{~b}}$ \\
PIB & 298 & $\mathrm{n} / \mathrm{a}$ & $3.85 \times 10^{-8}$ & $4.47 \times 10^{-8 \mathrm{c}}$ \\
PS & 298 & $2.24 \times 10^{-8}$ & $1.56 \times 10^{-8}$ & $3.02 \times 10^{-8 \mathrm{~d}}$ \\
1,4-PBD & 442.5 & $1.12 \times 10^{-10}$ & $9.95 \times 10^{-11}$ & 1.25 \\
\hline
\end{tabular}

${ }^{\text {a}}$ Pearson et al. 1987

${ }^{\mathrm{b}}$ Extracted from Fig. 2 of Haley et al. (2003)

${ }^{\mathrm{c}}$ Table 12-II of Ferry (1980)

literature. If possible, the magnitude of $\zeta$ is taken from measurements of the self-diffusion coefficient or viscosity of linear, non-entangled polymer melts, which are correctly described by the Rouse model. Contrary to linear, entangled polymer melts the influence of tube length fluctuations, tube dilation and constraint release are not of importance for linear, non-entangled polymer melts.

The $\tau_{\mathrm{e}}$ and $\zeta$ are related to each other via the relationship (Larson et al. 2003)

$\tau_{\mathrm{e}}=\left(\frac{M_{\mathrm{e}}^{G}}{M_{0}}\right)^{2} \frac{\zeta b^{2}}{3 \pi^{2} k_{B} T}=\left(\frac{M_{\mathrm{e}}^{G}}{M_{0}}\right) \frac{\zeta M_{\mathrm{e}}^{G}\left[\left\langle R^{2}\right\rangle_{0} / M\right]}{3 \pi^{2} k_{B} T}$,

with $M_{0}$ the monomer molecular weight, $b$ the monomer based segment length and $\left\langle R^{2}\right\rangle_{0}$ the unperturbed mean square end-to-end distance. The magnitude of $\zeta$, following from $\tau_{\mathrm{e}}^{\mathrm{LM}}$, using procedure $\mathrm{A}$ and $\mathrm{B}$ of the $\mathrm{LM}$ model are given in Table 4. For polyisobutylene, PIB, the magnitude of $\tau_{\mathrm{e}}^{\mathrm{LM}}$ at $T=298 \mathrm{~K}$ is obtained from procedure $\mathrm{B}$ of the $\mathrm{LM}$ model and using the expression for $\eta_{0}\left(M_{w}\right)$ reported by Fetters et al. (1991). Taking $G_{N}^{0}=330 \mathrm{kPa}$ and $M_{\mathrm{e}}^{G}=5700 \mathrm{~g} / \mathrm{mol}$ (Fetters et al. 1994) this gives $\tau_{\mathrm{e}}^{\mathrm{LM}}=1.05 \times 10^{-3} \mathrm{~s}$ at $T=298 \mathrm{~K}$. Observation of Table 4 reveals that the magnitude of $\zeta$ following from procedure $\mathrm{A}$ and $\mathrm{B}$ of the $\mathrm{LM}$ model are in agreement with the values reported in the literature. Hence, this demonstrates that a good estimate of $\tau_{\mathrm{e}}$, or $\zeta$, can be obtained using procedure $\mathrm{A}$, or $\mathrm{B}$, of the $\mathrm{LM}$ model.

\section{Determination of the equilibration time and monomer friction coefficient for isotactic and atactic polypropylene}

In the previous section it is shown that, for different polymer systems, procedure B of the LM model is suitable to obtain the magnitude of $\tau_{\mathrm{e}}$, or $\zeta$. Therefore this procedure can be applied to determine $\tau_{\mathrm{e}}$ for iPP. Procedure A cannot be followed due to the small temperature window between the temperature of crystallization and degradation in combination with the
${ }^{\mathrm{d}}$ Majeste et al. (1998); Pattamaprom et al. (2000)

${ }^{\mathrm{e}}$ Table 12-II of Ferry (1980) for rubbers

Table 5 The magnitude of the plateau modulus $G_{N}^{0}$, the molecular weight per entanglement, $M_{\mathrm{e}}^{G}$, at a given absolute temperature $T$, reported in the literature for polydisperse aPP and iPP melts

\begin{tabular}{lllll}
\hline Material & $T[\mathrm{~K}]$ & $G_{N}^{0}[\mathrm{kPa}]$ & $M_{\mathrm{e}}^{G}[\mathrm{~g} / \mathrm{mol}]$ & Reference \\
\hline aPP & 296 & 452 & 3720 & $\begin{array}{l}\text { Plazek and } \\
\text { Plazek (1983) }\end{array}$ \\
aPP & 463 & $480-600$ & $3900-4905$ & Vega et al. (2003) \\
aPP & 463 & 410 & 5640 & Eckstein et al. (1998) \\
iPP & 463 & 430 & 5500 & Eckstein et al. (1998) \\
\hline
\end{tabular}

difficulties to synthesize a melt of long nearly monodisperse iPP chains (Eckstein et al. 1998). In order to determine $\tau_{\mathrm{e}}$ the magnitude of $M_{\mathrm{e}}^{G}$, or $G_{N}^{0}$, is required, which can be obtained in three different ways.

First, the plateau modulus, $G_{N}^{0}$, is determined experimentally and $M_{\mathrm{e}}^{G}$ follows from Eq. (3). For monodisperse aPP melts at $T=348 \mathrm{~K}, G_{N}^{0}=480 \mathrm{kPa}$ and $M_{\mathrm{e}}^{G}=4000 \mathrm{~g} /$ mol, which follow from a linear interpolation between the reported experimental values at $T=298 \mathrm{~K}$ and $T=413 \mathrm{~K}$ in Fetters et al. (1994). For monodisperse iPP no data are reported. The magnitudes of $G_{N}^{0}$ and $M_{\mathrm{e}}^{G}$ reported in the literature for polydisperse aPP and iPP melts are given in Table 5. For aPP these values are in agreement with those of monodisperse aPP melts.

Second, the magnitude of $G_{N}^{0}$ and $M_{\mathrm{e}}^{G}$ can be determined from the relationships derived by Fetters et al. (1994, 1996, 1999), based on the concept of the packing length, $p$. The expressions for $p$ and $M_{\mathrm{e}}^{G}$ are (Fetters et al. 1994)

$p=\frac{M}{\left\langle R^{2}\right\rangle_{0}} \frac{1}{\rho N_{\mathrm{A}}}$,

$M_{\mathrm{e}}^{G}=B^{2} \rho N_{\mathrm{A}} \rho^{3}$,

with $B$ a temperature dependent constant, which equals 0.0516 at $T=413 \mathrm{~K}$ and 0.0565 at $T=298 \mathrm{~K}$ (Fetters et al. 1994). $G_{N}^{0}$ subsequently follows from Eq. (3). (For further details the reader is referred to the original paper.) It should be noted that the magnitude of $M_{\mathrm{e}}^{G}$, following from Eq. (9), is in good agreement with the 
Table 6 The magnitude of the plateau modulus $G_{N}^{0}$, the molecular weight per entanglement, $M_{\mathrm{e}}^{G}$, the equilibration time, $\tau_{\mathrm{e}}$, and the monomeric friction coefficient, $\zeta$, for iPP and aPP based on procedure B of the model of Likhtman and McLeish (2002), LM. The magnitude of $<R^{2}>_{0} / M$ is taken from Fetters et al. (1994) and Zirkel et al. (1992) for aPP and from Fetters et al. (1999) and Zirkel et al. (1992) for iPP

\begin{tabular}{llllllll}
$\begin{array}{l}\text { Material } \\
\text { Units }\end{array}$ & $T$ & $\mathrm{~K}$ & $\begin{array}{l}M_{\mathrm{e}}^{G} \\
\mathrm{~g} / \mathrm{mol}\end{array}$ & $\begin{array}{l}G_{N}^{0} \\
\mathrm{kPa}\end{array}$ & $\begin{array}{l}\tau_{\mathrm{e}} \\
\mathrm{s}\end{array}$ & $\begin{array}{l}\zeta \\
\mathrm{Ns} / \mathrm{m}\end{array}$ & $\begin{array}{l}<\mathrm{R}^{2}>{ }_{0} / \mathrm{M} \\
\AA^{2} \mathrm{~mol} / \mathrm{g}\end{array}$ \\
\hline aPP & 348 & 4000 & 470 & $1.60 \times 10^{-5}$ & $4.51 \times 10^{-10}$ & 0.664 & Fetters et al. (1994) \\
aPP & 348 & 3150 & 606 & $5.50 \times 10^{-6}$ & $2.51 \times 10^{-10}$ & 0.664 & LM \\
iPP & 463 & 5500 & 430 & $9.87 \times 10^{-8}$ & $1.86 \times 10^{-12}$ & 0.694 & Fetters et al. (1999) \\
iPP & 463 & 4400 & 538 & $3.54 \times 10^{-8}$ & $1.04 \times 10^{-12}$ & 0.694 & LM \\
\hline
\end{tabular}

experimental value for monodisperse melts of many different polymers (Fetters et al. 1994, 1996, 1999). For iPP and aPP melts the density and the magnitude of $<R^{2}>_{0} / M$, as determined by SANS measurement of iPP and aPP chains in the melt, are nearly identical (Zirkel et al. 1992; Schweizer et al. 1995; Eckstein et al. 1998; Fetters et al. 1999; Krishamoorti et al. 2002). As the parameters in Eqs. (8) and (9) are nearly identical for iPP and aPP melts, this equally holds for $M_{\mathrm{e}}^{G}$. The approximately identical values of iPP and aPP is confirmed by experiments (Eckstein et al. 1998), see Table 5, which are in good agreement with the predicted value from Eq. (9) (see Table 2 of Fetters et al. 1999).

Third, one may obtain a magnitude of $M_{\mathrm{e}}^{G}$ from the description of $G^{\prime}(\omega)$ and $G^{\prime \prime}(\omega)$ by procedure A of the LM model. For PI, PS and h-PBD the magnitude of $M_{\mathrm{e}}^{G}$ based on this approach is identical to the experimental values for monodisperse melts, which are in good agreement with the predictions from Eq. (9). However, for nearly monodisperse aPP melts at $T=348 \mathrm{~K}$ a good description of $G^{\prime}(\omega)$ and $G^{\prime \prime}(\omega)$ (Pearson et al. 1988) by the LM model is obtained provided $M_{\mathrm{e}}^{G}=3150 \mathrm{~g} / \mathrm{mol}$. This magnitude is a factor 1.25 smaller compared to the experimental value for monodisperse aPP melts, $M_{\mathrm{e}}^{G}=4000 \mathrm{~g} / \mathrm{mol}$, reported by Fetters et al. $(1994,1996)$.

The variation in the magnitude of $M_{\mathrm{e}}^{G}$ is unsatisfactory because $\eta_{0}$ scales with $M_{\mathrm{e}}^{G}$ as $\eta_{0} \sim \tau_{\mathrm{e}}^{\mathrm{LM}} G_{N}^{0}\left(M / M_{\mathrm{e}}^{G}\right)^{3.4} \sim \tau_{\mathrm{e}}^{\mathrm{LM}} M_{\mathrm{e}}^{G-4.4}$, and consequently $\tau_{\mathrm{e}}^{\mathrm{LM}} \sim \eta_{0} M_{\mathrm{e}}^{G 4.4}$. Hence, a small variation in $M_{\mathrm{e}}^{G}$ can have a strong effect on the estimated magnitude of $\tau_{\mathrm{e}}^{\mathrm{LM}}$. Therefore two values of $M_{\mathrm{e}}^{G}$ and $G_{N}^{0}\left(=4 G^{\mathrm{LM}} / 5\right)$ are considered for the iPP and aPP melts in the remaining of this section.

For aPP at $T=348 \mathrm{~K}$ the first set are the experimental values for the monodisperse melts reported by Fetters et al. (1994, 1996), $G_{N}^{0}=470 \mathrm{kPa}$ and $M_{\mathrm{e}}^{G}=4000 \mathrm{~g} / \mathrm{mol}$, and the second set are those following from the description of $G^{\prime}(\omega)$ and $G^{\prime \prime}(\omega)$ by the LM model, $G_{N}^{0}=606 \mathrm{kPa}$ and $M_{\mathrm{e}}^{G}=3150 \mathrm{~g} / \mathrm{mol}$. Application of procedure $\mathrm{B}$ of the LM model gives $\tau_{\mathrm{e}}^{\mathrm{LM}}=1.60 \times 10^{-5} \mathrm{~s}$ for $M_{\mathrm{e}}^{G}=4000 \mathrm{~g} / \mathrm{mol}$ and $\tau_{\mathrm{e}}^{\mathrm{LM}}=5.50 \times 10^{-6} \mathrm{~s}$ for $M_{\mathrm{e}}^{G}=3150 \mathrm{~g} / \mathrm{mol}$, respectively. Using Eq. (7) one obtains $\zeta=4.51 \times 10^{-10} \mathrm{~s}$ for $M_{\mathrm{e}}^{G}=4000 \mathrm{~g} / \mathrm{mol}$ and $\zeta=2.51 \times 10^{-10} \mathrm{~s}$ for $M_{\mathrm{e}}^{G}=3150 \mathrm{~g} / \mathrm{mol}$, respectively.
For iPP at $T=463 \mathrm{~K}$ the reported experimental values equal $G_{N}^{0}=430 \mathrm{kPa}$ and $M_{\mathrm{e}}^{G}=5500 \mathrm{~g} / \mathrm{mol}$ (Eckstein et al. 1998; Fetters et al. 1999). Maintaining a ratio of 1.25 between the experimental value and that following from the LM model, as observed for aPP, the second parameter set is equal to $G_{N}^{0}=538 \mathrm{kPa}$ and $M_{\mathrm{e}}^{G}=4400 \mathrm{~g} / \mathrm{mol}$. For iPP at $T=463 \mathrm{~K}$ this gives $\tau_{\mathrm{e}}^{\mathrm{LM}}=9.87 \times 10^{-8} \mathrm{~s} \quad$ for $\quad M_{\mathrm{e}}^{G}=5500 \mathrm{~g} / \mathrm{mol} \quad$ and $\tau_{\mathrm{e}}^{\mathrm{LM}}=3.54 \times 10^{-8} \mathrm{~s}$ for $M_{\mathrm{e}}^{G}=4400 \mathrm{~g} / \mathrm{mol}$, respectively. The magnitudes of $\zeta$ for iPP at $T=463 \mathrm{~K}$ are equal to $\zeta=1.86 \times 10^{-12} \mathrm{~s}$ for $M_{\mathrm{e}}^{G}=5500 \mathrm{~g} / \mathrm{mol}$ and $\zeta=1.04 \times 10^{-12} \mathrm{~s}$ for $M_{\mathrm{e}}^{G}=4400 \mathrm{~g} / \mathrm{mol}$, respectively. For aPP and iPP the magnitude of $\tau_{\mathrm{e}}$ and $\zeta$ following from the different sets of $M_{\mathrm{e}}^{G}$ and $G_{N}^{0}$ are summarized in Table 6. It should be noted that the difference in $\zeta$ is smaller compared to $\tau_{\mathrm{e}}^{\mathrm{LM}}$ as $\tau_{\mathrm{e}}^{\mathrm{LM}} \sim \eta_{0} M_{\mathrm{e}}^{G 4.4}$ whereas $\zeta \sim \eta_{0} M_{\mathrm{e}}^{G 2.4}$. Hence, the variation of $M_{\mathrm{e}}^{G}$ by a factor of 1.25 results into a difference of about a factor 2.9 for $\tau_{\mathrm{e}}^{\mathrm{LM}}$ and about a factor 1.8 for $\zeta$, respectively.

\section{Conclusion}

A procedure to estimate the equilibration time, $\tau_{\mathrm{e}}$, and the monomer friction coefficient, $\zeta$, from the zero shear rate viscosity, $\eta_{0}$, of linear, entangled polymer melts is given using the molecular weight per entanglement reported in the literature. For PE, h-PBD, 1.4-PBD, PS and aPP this gives an estimate of $\tau_{\mathrm{e}}$ which is in good agreement with that obtained from the description of $G^{\prime}(\omega)$ and $G^{\prime \prime}(\omega)$ using the molecular based model of Likhtman and McLeish (2002). The magnitude for $\zeta$ is also in good agreement with values reported in the literature for linear, nonentangled polymer melts of PE, PS, PIB and PI and that for a rubber of 1,4-PBD.

For aPP and dhhPP $G^{\prime}(\omega)$ and $G^{\prime \prime}(\omega)$ are correctly predicted by the LM model provided the molecular weight per entanglement, $M_{\mathrm{e}}^{G}$, is reduced by a factor 1.25 and 1.67, respectively, compared to the experimental value reported in the literature. Experimental results and the predicted magnitude of $M_{\mathrm{e}}^{G}$, based on the concept of the packing length (Eq. 9), are in good agreement with 
each other, and moreover indicate that the magnitude of $M_{\mathrm{e}}^{G}$ is approximately identical for aPP and iPP. Hence the question arises if one relies stronger on the experimental magnitude of $M_{\mathrm{e}}^{G}$ reported in the literature (Fetters et al. 1994, 1996, 1999) or that following from the description of $G^{\prime}(\omega)$ and $G^{\prime \prime}(\omega)$ using the model of Likhtmann and McLeish (2002). Finally, this difference in $M_{\mathrm{e}}^{G}$ results into a difference of about a factor 2.9 in the magnitude of $\tau_{\mathrm{e}}$ and about a factor 1.8 in $\zeta$ respectively; see Table 6. The discrepancy in the magnitude of $M_{\mathrm{e}}^{G}$ may be resolved by an analysis of $G^{\prime}(\omega)$ and $G^{\prime \prime}(\omega)$ using the LM model for a nearly monodisperse iPP or aPP melt where the magnitude of $G_{N}^{0} J_{e}^{0}$ has the usual value in the range 2.0-2.2.
In view of the goal to obtain a better understanding of flow induced crystallization experiments on iPP only the longest Rouse time of the high molecular weight chains, $\tau_{R}=\tau_{\mathrm{e}}\left(M / M_{\mathrm{e}}^{G}\right)^{2} \sim \zeta M^{2}$, is of interest. Here, one can partly benefit from the fact that a smaller $M_{\mathrm{e}}^{G}$ is balanced by a smaller $\tau_{\mathrm{e}}$. Finally, the difference in $\tau_{\mathrm{R}}$ is about a factor 1.8, which is acceptable for the purpose to obtain an estimate of the Deborah numbers for chain stretching the high molecular weight chains.

Acknowledgement The author thanks Prof. Hans Christian Öttinger for helpful discussions and comments on the manuscript.

\section{References}

Aguilar M, Vega JF, Sanz E, MarínezSalazar J (2001) New aspects on the rheological behavior of metallocene catalyzed polyethylenes. Polymer 42:97139721

Aguilar M, Vega JF, Pena B, MarínezSalazar J (2003) Novel features of the rheological behavior of metallocene catalyzed atactic polypropylene. Polymer 44:1401-1407

Berger L, Meissner J (1992) Linear viscoelasticity, simple and planar melt extension of linear polybutadienes with bimodal molar mass distributions. Rheol Acta 31:63-74

des Cloizeaux J (1988) Double reptation vs simple reptation in polymer melts. Europhys Lett 5:437-442

des Cloizeaux J (1990) Relaxation of entangled polymers in melts. Macromolecules 23:3392-4406

Doi M, Edwards SF (1986) The theory of polymer dynamics. Clarendon Press, Oxford

Eckstein A, Suhm J, Friedrich C, Maier RD, Sassmannshausen J, Bochmann M, Mühlhaupt R (1998) Determination of plateau moduli and entanglement molecular weights of isotactic, syndiotactic and atactic polypropylenes synthesized with metallocene catalysts. Macromolecules 31:1335-1340

Ferry JD (1980) Viscoelastic properties of polymers. Wiley, New York

Fetters LJ, Graessley WW, Kiss AD (1991) Viscoelastic properties of polyisobutylene melts. Macromolecules 24:3136-3141
Fetters LJ, Kiss AD, Pearson DS, Quack GF, Vitus FJ (1993) Rheological behavior of star-shaped polymers. Macromolecules 26:647-654

Fetters LJ, Lohse DJ, Richter D, Witten TA, Zirkel A (1994) Connection between polymer molecular weight, density, chain dimensions, and melt viscoelastic properties. Macromolecules 27:4639-4647

Fetters LJ, Lohse DJ, Colby R (1996) In: Mark JE (ed) Chain dimensions and entanglement spacings. Physical properties of polymers, pp 335-340

Fetters LJ, Lohse DJ, Graessley WW (1999) Chain dimensions and entanglements spacings in dense macromolecular systems. J Polym Sci B Polym Phys 37:1023-1033

Gell CB, Graessley WW, Letter LJ (1997) Viscoelasticity and self-diffusion in melts of entangled linear polymers. J Polym Sci B Polym Phys 35:1933-1942

Graessley WW, Roovers J (1979) Melt rheology of four-arm and six-arm star polystyrenes. Macromolecules 12:959965

Graham RS, McLeish TCB, Harlen OG (2001) Using the Pom-Pom equations to analyze polymer melts in exponential shear. J Rheol 45:275-290

Haley JC, Lodge TP, He Y, Ediger MD, von Meerwall ED, Mijovic J (2003) Composition and temperature dependence of terminal and segmental dynamics in polyisoprene/poly(vinylethylene) blends. Macromolecules 36:6142-6151

Keller A, Kolnaar HWH (1997) In: Meijer HEH (ed) Flow induced orientation and structure formation. Material Science and Technology 18:189-268

Kraft M, Meissner J, Kaschta J (1999) Linear viscoelastic characterization of polymer melts with long relaxation times. Macromolecules 32:751-757
Krishnamoorti R, Graessley WW, Zirkel A, Richter D, Hadjichristidis N, Letters LJ, Lohse DJ (2002) Melt-state polymer chain dimensions as a function of temperature. J Polym Sci B Polym Phys 40:1768-1776

Larson RG, Sridhar T, Leal LG, McKinley GH, Likhtman AE, McLeish TCB (2003) Definitions of entanglement spacing and time constants in the tube model. J Rheol 47:809-818

Likhtman AE, McLeish TCB (2002) Quantitative theory for linear dynamics of linear entangled polymers. Macromolecules 35:6332-6343

Majeste JC, Monfort JP, Allan A, Marin G (1998) Viscoelasticity of low molecular weight polymers and the transition to the entangled regime. Rheol Acta 37:486-499

Milner ST, McLeish TCB (1998) Reptation and contour length fluctuations in melts of linear polymers. Phys Rev Lett 81:725728

Nogales A, Hsiao BS, Somani RHM, Srinivas S, Tsou AH, Balta-Calleja FJ, Eqyuerra TA (2001) Shear-induced crystallization of isotactic polypropylene with different molecular weight distributions: in situ small- and wide-angle X-ray scattering studies. Polymer 42:5247-5256

Pattamaprom C, Larson RG (2001) Predicting the linear viscoelastic properties of monodisperse and polydisperse polystyrenes and polyethylenes. Rheol Acta 40:516-532

Pattamaprom C, Larson RG, Van Dyke TJ (2000) Quantitative predictions of linear viscoelastic rheological properties of entangled polymers. Rheol Acta 39:517531 
Pearson DS, Ver Strate G, von Meerwall E, Schilling FC (1987) Viscosity and selfdiffusion coefficient of linear polyethylene. Macromolecules 20:1133-1141

Pearson DS, Fetters LJ, Younghouse LB, Mays JW (1988) Rheological properties of poly(1,2-dimethyl-1-butenylene) and model atactic polypropylene. Macromolecules 21:478-484

Plazek DL, Plazek DJ (1983) Viscoelastic behavior of atactic polypropylene. Macromolecules 16:1469-1475

Raju VR, Rachpudy H, Graessley WW (1979) Properties of amorphous and crystallizable hydrocarbon polymers. IV. Melt rheology of linear and star-branched hydrogenated polybutadiene. J Polym Sci Polym Phys Ed 17:1223-1235

Raju VR, Menezes EV, Marin G, Graessley WW, Fetters LJ (1981) Concentration and molecular weight dependence of viscoelastic properties of linear and star polymers. Macromolecules 14:1668-1676

Schweizer KS, David EF, Singh C, Curro JG, Rajasekaran JJ (1995) Structureproperty correlations of atomistic and course-grained models of polymer melts. Macromolecules 28:1528-1540
Seki M, Thurman D, Oberhauser J, Kornfield JA (2002) Shear-mediated crystallization of isotactic polypropylene. The role of long chain-long chain overlap. Macromolecules 35:2583-2594

Struglinski MJ, Graessley WW (1985) Effects of polydispersity on the linear viscoelastic properties of entangled polymers. 1. Experimental observations for binary mixtures of linear polybutadiene. Macromolecules 18:2630-2643

Tsenoglou C (1987) Viscoelasticity of binary homopolymer blends. ACS Polym Preprints 28:185-186

van Meerveld J, Peters GWM, Hütter M (2003) Towards a rheological classification of flow induced crystallization experiments of polymer melts. Preprint

Vega JF, Aguilar M, Marínez-Salazar J (2003) Model linear metallocene-catalyzed polyolefins: melt rheological behavior and molecular dynamics. $\mathbf{J}$ Rheol 47:1505-1521

Vleeshouwers S, Meijer HEH (1996) A rheological study of shear induced crystallization. Rheol Acta 35:391-399
Wasserman SH, Graessley WW (1992) Effects of polydispersity on linear viscoelasticity in entangled polymer melts. J Rheol 36:543-572

Wasserman SH, Graessley WW (1996) Prediction of linear viscoelastic response for entangled polyolefin melts from molecular weight distribution. Polym Eng Sci 36:852-861

Wood-Adams PM, Dealy JM,

de Groot AW, Redwine OD (2000) Effect of molecular structure on the linear viscoelastic behavior of polyethylene. Macromolecules 33:7489-7499

Zirkel A, Urban V, Richter D, Fetters LJ, Huang JS, Kampmann R, Hadjichristidis H (1992) Small-angle neutron scattering evaluation of the temperature dependence of atactic polypropylene and poly(1-butene) chain dimensions in the melt. Macromolecules 25:6148-6155 